\title{
Evaluación de las habilidades blandas en la educación superior
}

\author{
Gina Y. Fuentes ${ }^{1}$, Luís M. Moreno-Murcia ${ }^{2}$, Diana C. Rincón-Tellez ${ }^{1}$ y María B. Silva-Garcia ${ }^{2}$ \\ (1) Facultad de Ciencias Empresariales, Programa de Administración en Seguridad y Salud en el Trabajo y \\ Administración en Salud Ocupacional, Corporación Universitaria Minuto de Dios - UNIMINUTO. Zipaquirá-Colombia. \\ (Correo-e: gfuentesroj@uniminuto.edu.co; drincontell@uniminuto.edu.co) \\ (2) Facultad de Ciencias Humanas y Sociales, Unidad de Investigación-Programa de Psicología, Corporación \\ Universitaria Minuto de Dios - UNIMINUTO. Bogotá-Colombia. \\ (Correo-e:Iuis.moreno.mu@uniminuto.edu.co;maria.silva.ga@uniminuto.edu)
}

Recibido Dic. 22, 2020; Aceptado Feb. 22, 2021; Versión final Mar. 26, 2021, Publicado Ago. 2021

\begin{abstract}
Resumen
La presente investigación tiene como objetivo determinar la prevalencia de habilidades blandas en estudiantes de la Corporación Universitaria Minuto de Dios - UNIMINUTO (Colombia), de los programas académicos de psicología y administración en salud ocupacional de las sedes Bogotá sur y Zipaquirá. El enfoque es de tipo cuantitativo, con alcance descriptivo y corte transversal aplicado a una muestra de 50 estudiantes que cursan los últimos semestres. Se identifica que las habilidades blandas con mayor prevalencia en los estudiantes de los programas académicos estudiados son escucha, influencia y trabajo en equipo. Las habilidades que se deben fortalecer son responsabilidad y apoyo. Se concluye que el mercado laboral observa y exige la presencia de habilidades blandas en sus profesionales, siendo este un cambio y una oportunidad frente al entorno para que la academia a través de la articulación del currículo fortalezca las conductas asociadas a cada una de las habilidades.
\end{abstract}

Palabras clave: conductas; competencias; educación superior; empleabilidad; habilidades blandas

\section{Evaluation of soft skills in higher education}

\begin{abstract}
The objective of this research study is to determine the prevalence of soft skills in students at the Corporación Universitaria Minuto de Dios - UNIMINUTO (Colombia), in the academic programs of psychology and occupational health administration at the Bogotá-South and Zipaquirá campuses. The approach of the study is quantitative, descriptive in scope, and cross-sectional. The sample consists of 50 students in their last semesters. The results show that the most prevalent soft skills are listening, influence, and teamwork. The soft skills that require strengthening are responsibility and support. It is concluded that the labor market observes and demands the presence of soft skills in professionals, opening an opportunity for academics to strengthen behaviors associated with each soft skill by adapting academic curricula.
\end{abstract}




\section{INTRODUCCIÓN}

Las Soft Skills (habilidades blandas) son usualmente relacionadas con comportamientos, interacción con los demás y patrones de personalidad; este constructo se refiere a las capacidades aprendidas y utilizables para la adaptabilidad en los diferentes contextos en los que pueda relacionarse el sujeto, tales como su ambiente escolar o de empleabilidad (Cobo y Moravec, 2011). De modo que son aquellas habilidades, que permiten poner en práctica valores que contribuyen a que la persona se desarrolle adecuadamente en los distintos ámbitos de acción (Buxarrais, 2013), lo cual genera una diferenciación entre dos personas, así como la identificación de características tales como empatía, buenos modales, capacidad de negociación, espíritu de colaboración, puntualidad y optimismo (Espinosa y Gallegos, 2020).

Para Ortega (2016) las habilidades blandas o no cognitivas, se definen como las prácticas, actitudes y capacidades que tiene una persona para relacionarse e interactuar con el mundo. Estas permiten entre otros procesos; la comprensión de emociones, logro de objetivos, toma de decisiones y la capacidad de afrontar situaciones adversas, el autor resalta la interacción que se debe tener con el otro. De la misma manera, Ortega et al. (2016) definen las habilidades blandas como capacidades adaptativas y comportamentales, que permiten a las personas interactuar en contextos socio-históricos y culturales, de tal forma que respondan a las necesidades y demandas del mismo; tienen un impacto en la vida de las personas, por lo tanto, se considera sumamente importante su desarrollo desde edades tempranas.

En la actualidad los procesos de selección están enmarcados en un modelo de gestión por competencias, en el que se busca conocer las habilidades, aptitudes, actitudes y conocimientos de la persona, con el fin de contratar al mejor candidato (Kalugina y Shvydun, 2014). Es así como las habilidades blandas y duras toman gran relevancia en el ámbito organizacional, en donde los egresados de la educación superior buscan generar un impacto positivo. Las habilidades blandas están relacionadas con las experiencias previas desde la infancia y la adolescencia; según Ortega (2016) en estas etapas el proceso de aprendizaje se genera de manera más concreta, y pueden ser fortalecidas a través del proceso de escolarización (Berry y Routon, 2020), por lo tanto, se convierten en el pilar fundamental para ser utilizadas en los procesos de inmersión laboral, que exigen diferentes contextos empresariales (Cobo y Moravec, 2011).

A nivel internacional, se observa que el interés de los empresarios suele dirigirse a seleccionar personas con liderazgo, habilidad de comunicación, valores éticos, conocimiento y trabajo en equipo (Tito y Serrano, 2016), habilidades que fueron aprendidas desde la infancia, es decir que cada uno de los eslabones académicos permitieron fortalecerlas de tal manera que al llegar a la inmersión laboral estas sean evidentes. Sin embargo, Gómez (2019) enfatiza en una problemática educativa enmarcada en la falta de desarrollo de las habilidades no cognitivas en la educación superior, por lo tanto, dificultades en su empleabilidad, teniendo en cuenta que para la vinculación al mercado laboral exige en un $51 \%$ habilidades blandas, un $9 \%$ habilidades cognitivas avanzadas, un $16 \%$ habilidades técnicas y en un $4 \%$ habilidades cognitivas básicas.

Por lo cual, se evidencian falencias al modelo educativo actual, en algunas ocasiones basado en principios de producción industrial y manufacturera (Buxarrais, 2013) dejando a un lado el desarrollo y fortalecimiento de las habilidades sociales, las cuales deben considerarse como un eje transversal en los procesos de formación y capacitación al interior de los centros educativos, cuyo énfasis principal debería ser habilidades con principios éticos, que favorezcan escenarios de felicidad y libertad; y que también promuevan habilidades blandas como: el trabajo en equipo, el pensamiento crítico y analítico, comunicación asertiva, creatividad, empatía, resolución de conflictos, resiliencia entre otras, que garantizaran el éxito personal y laboral de los estudiantes (Tseng et al. 2019).

En la práctica, algunos autores coinciden que la falencia de estas habilidades que presentan los egresados de instituciones de educación superior, genera un obstáculo para responder a las necesidades actuales de los gremios empresariales, se centra como ya se mencionó en los procesos educativos que se imparten en la educación formal, en donde prima el aprendizaje de habilidades duras y en un segundo lugar permanecen las habilidades blandas. De aquí la importancia de fusionar los procesos educativos con los sectores productivos, por medio de estrategias como el coaching universitario, el cual se encuentra en implementación en algunas universidades al favorecer el desarrollo de competencias transversales mediante el empoderamiento y la consciencia de potencialidades personales (Musicco, 2018).

En este orden de ideas, siendo un tema de gran interés, un sin número de investigaciones se han realizado con el fin de identificar las habilidades blandas (Zhang, 2012) que se requieren en diferentes sectores económicos, se reconocen y contemplan generaciones que hoy en día reciben el nombre de millennials y centennials, quienes presentan diversas competencias como la capacidad creativa, capacidad de actualización, habilidad para trabajar en diferentes contextos internacionales que los convierten en llamativos ante la demanda laboral (Araya y González, 2019). De aquí la importancia de evaluar las competencias 
actuales que presentan los estudiantes en programas de educación superior, considerando las diferentes generaciones que se presentan en el ámbito académico y así poder establecer las estrategias formativas que orienten resultados adecuados al logro de los objetivos empresariales y personales, resolución de conflictos, toma de decisiones, inteligencia emocional y trabajo en equipo, competencias que buscan las empresas en los egresados para procesos de selección e inmersión laboral. En la actualidad se evidencia aún más, la necesidad de explorar y fortalecer las habilidades blandas a partir de las problemáticas que se presentan a nivel empresarial como efecto de la COVID-19, es así como Runciman (2021) manifiesta que se debe dar un cambio en las competencias de liderazgo, con tendencia al fortalecimiento de habilidades como la empatía, la narración de historias, la inteligencia emocional, la confianza y la gestión del tiempo, lo cual se ve reflejado en el manejo y conocimiento del cliente.

Es interesante observar cómo los diferentes estudios enfatizan la importancia de desarrollar y fortalecer las habilidades blandas en la educación, como característica indispensable que debe prevalecer en el plan de estudios de los diferentes programas académicos. Sin embargo, el primer paso es conocer el nivel de competencias y habilidades blandas en los estudiantes, dado que este es un aprendizaje que inicia desde temprana edad, pero no resulta suficiente para su empleabilidad, generando incertidumbre en su desarrollo ocupacional y experiencia laboral, que en algunas oportunidades incide en el éxito profesional y laboral. Con base en lo anterior, el objetivo de la presente investigación es establecer la prevalencia de habilidades blandas y la asociación existente en estudiantes de últimos semestres de la Corporación Universitaria Minuto de Dios - UNIMINUTO de los programas de Psicología y Administración en Salud Ocupacional.

\section{MÉTODO}

Para esta investigación se realizó un estudio con enfoque cuantitativo de corte transversal y alcance descriptivo, aplicado a una muestra por conveniencia de 50 estudiantes de UNIMINUTO, distribuidos homogéneamente en dos programas académicos (Psicología y Administración en Salud Ocupacional) de las sedes Bogotá Sur y Zipaquirá respectivamente, teniendo en cuenta las siguientes condiciones: consentimiento informado voluntario, estar cursando el semestre octavo, noveno o décimo. Se realiza una caracterización y el análisis de conductas a los estudiantes que participaron en el estudio; la caracterización de la muestra se desarrolló aplicando una encuesta, en la cual se incluyeron los siguientes aspectos: sexo, edad, estrato, ciudad de residencia, estado civil, carrera que cursa, semestre, año de ingreso.

La determinación de habilidades blandas (escucha, habilidad para plantear preguntas, presentación, autoconfianza e Influencia), se realizó a través de dos ejercicios interactivos que se diseñaron a partir de la investigación realizada por Groh et al. (2015). El primer ejercicio consistió en un juego de roles que coloca al sujeto de estudio bajo presión; el estudiante asumió el rol de un asesor de servicio al cliente, uno de los investigadores desempeñó el rol de cliente molesto y otro investigador cumplió con la función de observador y evaluador de las conductas asociadas a cada una de las habilidades categorizadas como se observa en la tabla 1.

Tabla 1: Habilidades y conductas observables (categorizadas) en técnica de juego de roles

\begin{tabular}{|c|c|c|}
\hline Habilidad & Conductas observables & Categoría \\
\hline \multirow{5}{*}{ Escucha } & La conducta no verbal facial demuestra sinceridad. & 1 \\
\hline & Adopta postura de apertura. & 2 \\
\hline & Postura corporal se dirige al equipo. & 3 \\
\hline & Establece contacto visual. & 4 \\
\hline & Permite que los integrantes manifiesten sus opiniones sin interrumpirlos. & 5 \\
\hline \multirow{5}{*}{$\begin{array}{c}\text { Habilidad para } \\
\text { plantear } \\
\text { preguntas }\end{array}$} & Realiza preguntas de seguimiento-follow up (encadenadas) & 1 \\
\hline & Realiza preguntas abiertas & 2 \\
\hline & Utiliza preguntas No dirigidas & 3 \\
\hline & Utiliza preguntas de clarificación & 4 \\
\hline & Utiliza un estilo no amenazante & 5 \\
\hline \multirow{5}{*}{ Presentación } & Utiliza un tono de voz claro & 1 \\
\hline & Mantiene contacto visual & 2 \\
\hline & Habla de manera concisa & 3 \\
\hline & Expresa pensamientos a través del lenguaje no verbal & 4 \\
\hline & Realiza transiciones progresivas de un tema a otro. & 5 \\
\hline \multirow{5}{*}{ Autoconfianza } & Habla & 1 \\
\hline & No divaga- lógica y argumentación & 2 \\
\hline & Defiende su opinión mediante un estilo de comunicación no agresivo & 3 \\
\hline & Entrega mensajes y opiniones mediante de manera firme & 4 \\
\hline & Pregunta a los participantes para dar ejemplos. & 5 \\
\hline
\end{tabular}


Tabla 1: continuación

\begin{tabular}{|c|l|c|}
\hline Habilidad & Conductas observables & Categoría \\
\hline \multirow{4}{*}{ Influencia } & Presenta argumentos de diferentes maneras & 1 \\
\cline { 2 - 3 } & Busca establecer puntos de acuerdo con otros & 2 \\
\cline { 2 - 3 } & Dirige la discusión utilizando un estilo no agresivo. & 3 \\
\cline { 2 - 3 } & Manifiesta sus opiniones y aclara la forma en que estas pueden beneficiar a otros. & 4 \\
\cline { 2 - 3 } & Está preparado para comprometerse con el logro de acuerdos mutuos. & 5 \\
\hline
\end{tabular}

El segundo ejercicio consistió en una entrevista individual por competencias, en la cual se aplicó un cuestionario de 10 preguntas, se observó atentamente a cada uno de los participantes durante la ejecución del ejercicio y se realizó la evaluación a través de una lista de chequeo, para identificar conductas relacionadas con las habilidades blandas de liderazgo, conciliación, iniciativa, organización y trabajo en equipo, que se categorizan y evidencian en la tabla 2.

Tabla 2: Habilidades y conductas observables (categorizadas) en técnica de entrevista individual a estudiantes

\begin{tabular}{|c|c|c|}
\hline Habilidad & Conductas observables & Categoría \\
\hline \multirow{5}{*}{ Liderazgo } & Se muestra activo en la discusión y provee puntos efectivos. & 1 \\
\hline & Expresa confianza mediante estilos de comunicación no agresivos. & 2 \\
\hline & Crea un ambiente positivo para el trabajo & 3 \\
\hline & Orientado al logro & 4 \\
\hline & Intenta tomar decisiones & 5 \\
\hline \multirow{5}{*}{ Conciliación } & Pregunta a participantes no activos acerca de sus opiniones & 1 \\
\hline & Intenta dirigir y coordinar la discusión de manera efectiva & 2 \\
\hline & $\begin{array}{l}\text { Resuelve el conflicto entre los integrantes del equipo utilizando un estilo } \\
\text { diplomático }\end{array}$ & 3 \\
\hline & Reconoce las contribuciones de otros. & 4 \\
\hline & Motiva a los demás integrantes a aportar más ideas. & 5 \\
\hline \multirow{5}{*}{ Iniciativa } & Genera buena impresión en otros. & 1 \\
\hline & Motiva al equipo a pensar en el futuro. & 2 \\
\hline & Propone ideas de alta calidad. & 3 \\
\hline & Es voluntario para realizar tareas no deseadas. & 4 \\
\hline & Motiva al grupo a lograr los objetivos. & 5 \\
\hline \multirow{5}{*}{ Organización } & Maneja el tiempo de discusión de manera efectiva & 1 \\
\hline & Recuerda a los demás frente a los tiempos establecidos. & 2 \\
\hline & Establece tareas para si mismo y se compromete a lograrla hasta el final. & 3 \\
\hline & $\begin{array}{l}\text { Solicita a otros centrarse cuando se han distanciado del punto central de la } \\
\text { discusión. }\end{array}$ & 4 \\
\hline & Define problemas y establece métodos para resolverlos. & 5 \\
\hline \multirow{4}{*}{ Trabajo en equipo } & Construye buenas relaciones con otros integrantes del equipo. & 1 \\
\hline & $\begin{array}{l}\text { Es tolerante con los miembros del equipo y acepta sus solicitudes o } \\
\text { inquietudes. }\end{array}$ & 2 \\
\hline & Da información acerca de la manera más adecuada para resolver la tarea. & 3 \\
\hline & Trabaja para resolver la tarea y está involucrado completamente en ella. & 4 \\
\hline
\end{tabular}

Tanto para el ejercicio de juego de roles como de entrevista individual por competencias, se utilizó como instrumento de recolección de datos una lista de chequeo con una escala de medición de tipo ordinal dicotómica, ya que se establece una prioridad en la evaluación, siendo 1 la calificación para la presencia de la conducta en el participante y 0 si no la presenta. Para medir la confiabilidad interna de los instrumentos usados para la recolección de la información en los dos ejercicios, se utilizó el estadístico Kuder-Richardson (tabla 3), por tratarse de una escala dicotómica, el KR-20 fue de 0,74 y 0,79 respectivamente, considerándose una categoría alta, lo cual significa que los instrumentos son confiables y cuentan con consistencia interna.

Tabla 3: Prueba de confiabilidad

\begin{tabular}{|l|c|c|}
\hline \multicolumn{3}{|c|}{ Estadístico de confiabilidad } \\
\hline Ejercicio & K de elementos & Kuder-Richardson (KR-20) \\
\hline Juego de roles & 25 & 0,74 \\
\hline Encuesta individual de competencias & 24 & 0,79 \\
\hline
\end{tabular}




\section{RESULTADOS}

La investigación se desarrolló siguiendo las etapas que se mencionan a continuación: determinación de la población y muestra, elaboración de los instrumentos, aplicación de los ejercicios a la muestra seleccionada, organización y tabulación de la información, análisis de datos cuantitativamente, haciendo uso de estadística descriptiva.

El análisis de la información general suministrada por los estudiantes contiene elementos como sexo, estrato, estado civil, semestre que cursa el estudiante, el año de ingreso a la carrera, estado laboral y tiempo de dedicación a sus actividades académicas, entre otras variables; por ser la investigación de tipo cuantitativo en la tabla 4 se observa la distribución de frecuencias de cada una de las variables evaluadas, para caracterizar la muestra seleccionada que corresponde a 50 estudiantes, 25 de ellos del programa académico de Psicología y 25 del programa académico de Administración en Salud Ocupacional.

Tabla 4: Información General de los Estudiantes

\begin{tabular}{|c|c|c|c|c|}
\hline \multirow{2}{*}{$\begin{array}{l}\text { Programa Académico } \\
\text { Variables }\end{array}$} & \multicolumn{2}{|c|}{ Administración en Salud Ocupacional } & \multicolumn{2}{|c|}{ Psicología } \\
\hline & \multirow{2}{*}{$n$} & \multirow{2}{*}{$\%$} & \multirow{2}{*}{$n$} & \multirow{2}{*}{$\%$} \\
\hline Género & & & & \\
\hline Masculino (1) & 10 & $42 \%$ & 5 & $20 \%$ \\
\hline Femenino (2) & 14 & $58 \%$ & 20 & $80 \%$ \\
\hline \multicolumn{5}{|l|}{ Semestre } \\
\hline Octavo (1) & 8 & $32 \%$ & 9 & $36 \%$ \\
\hline Noveno (2) & 8 & $32 \%$ & 6 & $24 \%$ \\
\hline Décimo (3) & 9 & $36 \%$ & 10 & $40 \%$ \\
\hline \multicolumn{5}{|l|}{ Estado Civil } \\
\hline Casado (1) & 4 & $16 \%$ & 5 & $20 \%$ \\
\hline Soltero (2) & 16 & $64 \%$ & 13 & $52 \%$ \\
\hline Unión Libre (3) & 5 & $20 \%$ & 6 & $24 \%$ \\
\hline Separado (4) & 0 & $0 \%$ & 1 & $4 \%$ \\
\hline \multicolumn{5}{|l|}{ Edad } \\
\hline Menos de $20(1)$ & 0 & $0 \%$ & 0 & $0 \%$ \\
\hline Entre 21 - $30(2)$ & 14 & $56 \%$ & 15 & $60 \%$ \\
\hline Entre 31 - $40(3)$ & 11 & $44 \%$ & 8 & $32 \%$ \\
\hline Entre $41-50(4)$ & 0 & $0 \%$ & 1 & $4 \%$ \\
\hline Más de $50(5)$ & 0 & $0 \%$ & 1 & $4 \%$ \\
\hline $\mathrm{NR}(0)$ & 0 & $0 \%$ & 0 & $0 \%$ \\
\hline \multicolumn{5}{|c|}{ Formación Académica Adicional } \\
\hline Ninguna (0) & 6 & $24 \%$ & 4 & $16 \%$ \\
\hline Técnico (1) & 13 & $52 \%$ & 11 & $44 \%$ \\
\hline Tecnológico (2) & 4 & $16 \%$ & 3 & $12 \%$ \\
\hline Universitario (3) & 0 & $0 \%$ & 1 & $4 \%$ \\
\hline Postgrado (4) & 0 & $0 \%$ & 1 & $4 \%$ \\
\hline Formación Continua (5) & 2 & $8 \%$ & 5 & $20 \%$ \\
\hline \multicolumn{5}{|l|}{ Actividad Laboral } \\
\hline Ninguna (0) & 2 & $8 \%$ & 0 & $0 \%$ \\
\hline Empleado (1) & 23 & $92 \%$ & 12 & $48 \%$ \\
\hline Desempleado (2) & 0 & $0 \%$ & 5 & $20 \%$ \\
\hline Actividades del Hogar (3) & 0 & $0 \%$ & 8 & $32 \%$ \\
\hline \multicolumn{5}{|l|}{ Nivel de Estrato } \\
\hline Uno (1) & 4 & $16 \%$ & 2 & $8 \%$ \\
\hline Dos (2) & 12 & $48 \%$ & 11 & $44 \%$ \\
\hline Tres (3) & 9 & $36 \%$ & 11 & $44 \%$ \\
\hline Cuatro (4) & 0 & $0 \%$ & \begin{tabular}{|c|}
1 \\
\end{tabular} & $4 \%$ \\
\hline Cinco (5) & 0 & $0 \%$ & 0 & $0 \%$ \\
\hline Seis (6) & 0 & $0 \%$ & 0 & $0 \%$ \\
\hline \multicolumn{5}{|l|}{ Ha Trabajado } \\
\hline Si (1) & 24 & $96 \%$ & 23 & $92 \%$ \\
\hline No $(2)$ & 1 & $4 \%$ & 2 & $8 \%$ \\
\hline \multicolumn{5}{|c|}{ Dedicación Académica Diaria } \\
\hline De 1 a 3 horas (1) & 16 & $64 \%$ & 8 & $32 \%$ \\
\hline De 4 a 6 horas (2) & 9 & $36 \%$ & 11 & $44 \%$ \\
\hline De 7 a 10 horas (3) & 0 & $0 \%$ & 6 & $24 \%$ \\
\hline Más de 10 horas (4) & 0 & $0 \%$ & 0 & $0 \%$ \\
\hline
\end{tabular}


Se evidencia la prevalencia del género femenino en el programa de psicología con un $80 \%$, en comparación con el programa de administración en salud ocupacional, donde se evidencia una distribución casi homogénea en cuanto al género que participó en la entrevista, es decir, que se tiene una participación del $58 \%$ de género femenino y el $42 \%$ del género masculino; sin embargo, prevalece el género femenino por una mínima diferencia. Con relación al estado civil, se encuentra que los participantes del estudio refieren con mayor frecuencia estar solteros con un $64 \%$ y $52 \%$; la edad promedio se encuentra entre los 21 y 30 años, con un $56 \%$ y $60 \%$ en los programas de administración en salud ocupacional y psicología respectivamente.

Es de resaltar que dentro de la caracterización de la población entrevistada, prevalece la formación académica técnica, tanto en el programa de administración en salud ocupacional, como en el programa de psicología; así mismo, se evidencia que la mayoría de los estudiantes son empleados, en el programa de psicología se encuentran estudiantes dedicados a realizar actividades del hogar, mientras que en el programa de administración en salud ocupacional no existen estudiantes realizando este tipo de actividad, aquí se evidencia una diferencia en cuanto empleabilidad entre los dos programas, ya que tan sólo el $48 \%$ de los estudiantes del programa de psicología se encuentran empleados en comparación con un $92 \%$ del programa de administración en salud ocupacional.

El $24 \%$ de los estudiantes del programa de psicología le dedican de 7 a 10 horas diarias a sus actividades académicas, no se evidencia dedicación de más de 10 horas por parte de los estudiantes de ambos programas; y en el caso de los estudiantes de administración en salud ocupacional, se evidencia una dedicación mínima, es decir de una a tres horas diarias por parte de los estudiantes a sus actividades académicas, lo cual corresponde a un $64 \%$ de la muestra.

En la ejercicio realizado a través del juego de roles, se evalúan conductas relacionadas con habilidades blandas categorizadas como se menciona en la tabla 5 , se realiza un análisis de las medidas de tendencia central (media, mediana y moda) y se observa también el análisis de medidas de dispersión (varianza y desviación estándar) para cada conducta. Con relación a las medidas de tendencia central, se evidencia que son homogéneas para las habilidades blandas de influencia y escucha, es decir, que los estudiantes de ambos programas presentan conductas, que permiten definir la presencia de la mismas, sin embargo, los estudiantes de ambos programas, no cuentan con conductas que permitan evaluar la habilidad para plantear preguntas, específicamente criterios cómo realizar preguntas de seguimiento que estén encadenadas, realizar preguntas abiertas, utilizar preguntas no dirigidas y utilizar preguntas de clarificación.

Tabla 5: Medidas de Tendencia Central de las habilidades evaluadas en juego de roles *Programa de Psicología ** Programa de Administración en Salud Ocupacional

\begin{tabular}{|c|c|c|c|c|c|c|c|}
\hline \multirow{2}{*}{ Habilidad } & \multirow{2}{*}{ Conducta } & \multicolumn{2}{|c|}{ Media } & \multicolumn{2}{|c|}{ Mediana } & \multicolumn{2}{|c|}{ Moda } \\
\hline & & $1^{*}$ & $2^{* *}$ & $1^{*}$ & $2^{* \star}$ & $1^{*}$ & $2^{* *}$ \\
\hline \multirow{5}{*}{ Escucha } & 1 & 1 & 1 & 1 & 1 & 1 & 1 \\
\hline & 2 & 1 & 1 & 1 & 1 & 1 & 1 \\
\hline & 3 & 1 & 1 & 1 & 1 & 1 & 1 \\
\hline & 4 & 1 & 1 & 1 & 1 & 1 & 1 \\
\hline & 5 & 1 & 1 & 1 & 1 & 1 & 1 \\
\hline \multirow{5}{*}{$\begin{array}{l}\text { Habilidad para } \\
\text { plantear preguntas }\end{array}$} & 1 & 0 & 0 & 0 & 0 & 0 & 0 \\
\hline & 2 & 0 & 0 & 0 & 0 & 0 & 0 \\
\hline & 3 & 0 & 0 & 0 & 0 & 0 & 0 \\
\hline & 4 & 1 & 0 & 1 & 0 & 1 & 0 \\
\hline & 5 & 1 & 1 & 1 & 1 & 1 & 1 \\
\hline \multirow[t]{5}{*}{ Presentación } & 1 & 1 & 1 & 1 & 1 & 1 & 1 \\
\hline & 2 & 1 & 1 & 1 & 1 & 1 & 1 \\
\hline & 3 & 1 & 1 & 1 & 1 & 1 & 1 \\
\hline & 4 & 0 & 1 & 0 & 1 & 0 & 1 \\
\hline & 5 & 0 & 0 & 0 & 0 & 0 & 0 \\
\hline \multirow{5}{*}{ Autoconfianza } & 1 & 1 & 1 & 1 & 1 & 1 & 1 \\
\hline & 2 & 1 & 1 & 1 & 1 & 1 & 1 \\
\hline & 3 & 1 & 1 & 1 & 1 & 1 & 1 \\
\hline & 4 & 1 & 1 & 1 & 1 & 1 & 1 \\
\hline & 5 & 0 & 0 & 0 & 0 & 0 & 0 \\
\hline \multirow{5}{*}{ Influencia } & 1 & 1 & 1 & 1 & 1 & 1 & 1 \\
\hline & 2 & 1 & 1 & 1 & 1 & 1 & 1 \\
\hline & 3 & 1 & 1 & 1 & 1 & 1 & 1 \\
\hline & 4 & 1 & 1 & 1 & 1 & 1 & 1 \\
\hline & 5 & 1 & 1 & 1 & 1 & 1 & 1 \\
\hline
\end{tabular}


En los estudiantes de psicología y administración en salud ocupacional se encuentra una similitud con relación a la habilidad de autoconfianza, esta similitud consiste en que ninguno de ellos mostró durante el juego de roles la conducta de preguntar a los participantes para dar ejemplos; en cuanto a la habilidad de presentación los estudiantes del programa de administración en salud ocupacional, utilizan un tono de voz claro, mantienen contacto visual, hablan de manera concisa y expresan pensamientos a través del lenguaje no verbal; mientras que los estudiantes de psicología expresan el uso de un tono de voz claro, mantienen contacto visual y hablan de manera concisa, pero, para el caso de los estudiantes de psicología, no se evidencian criterios como la expresión de pensamientos a través del lenguaje no verbal y la realización de transiciones progresivas de un tema otro; este último tampoco se evidencia en los estudiantes de administración en salud ocupacional. La desviación estándar permite verificar una homogeneidad en el resultado de la observación en el juego de roles frente a las conductas presentadas por cada estudiante con relación a cada habilidad, se evidencia una desviación estándar máxima de 0,5 y una mínima de 0,0, como se puede observar en la tabla 6.

Tabla 6: Medidas de dispersión de las habilidades evaluadas en juego de roles *Programa de Psicología ** Programa de Administración en Salud Ocupacional

\begin{tabular}{|c|c|c|c|c|c|}
\hline \multirow{2}{*}{ Habilidad } & \multirow{2}{*}{ Conducta } & \multicolumn{2}{|c|}{ Varianza } & \multicolumn{2}{|c|}{ Desviación Estándar } \\
\hline & & $1^{*}$ & $2^{* *}$ & $1^{*}$ & $2^{* *}$ \\
\hline \multirow{5}{*}{ Escucha } & 1 & 0,2 & 0,0 & 0,4 & 0,0 \\
\hline & 2 & 0,2 & 0,0 & 0,4 & 0,2 \\
\hline & 3 & 0,1 & 0,0 & 0,3 & 0,0 \\
\hline & 4 & 0,1 & 0,0 & 0,3 & 0,0 \\
\hline & 5 & 0,0 & 0,1 & 0,2 & 0,3 \\
\hline \multirow{5}{*}{$\begin{array}{l}\text { Habilidad para } \\
\text { hacer preguntas }\end{array}$} & 1 & 0,2 & 0,2 & 0,5 & 0,5 \\
\hline & 2 & 0,2 & 0,2 & 0,5 & 0,4 \\
\hline & 3 & 0,0 & 0,1 & 0,2 & 0,4 \\
\hline & 4 & 0,2 & 0,3 & 0,5 & 0,5 \\
\hline & 5 & 0,0 & 0,1 & 0,0 & 0,3 \\
\hline \multirow{5}{*}{ Presentación } & 1 & 0,0 & 0,0 & 0,0 & 0,2 \\
\hline & 2 & 0,3 & 0,0 & 0,5 & 0,0 \\
\hline & 3 & 0,1 & 0,1 & 0,3 & 0,3 \\
\hline & 4 & 0,2 & 0,1 & 0,5 & 0,3 \\
\hline & 5 & 0,2 & 0,3 & 0,5 & 0,5 \\
\hline \multirow{5}{*}{ Autoconfianza } & 1 & 0,0 & 0,0 & 0,0 & 0,0 \\
\hline & 2 & 0,2 & 0,2 & 0,5 & 0,5 \\
\hline & 3 & 0,0 & 0,0 & 0,0 & 0,2 \\
\hline & 4 & 0,1 & 0,1 & 0,3 & 0,3 \\
\hline & 5 & 0,0 & 0,2 & 0,2 & 0,4 \\
\hline \multirow{5}{*}{ Influencia } & 1 & 0,2 & 0,0 & 0,5 & 0,2 \\
\hline & 2 & 0,1 & 0,0 & 0,3 & 0,2 \\
\hline & 3 & 0,0 & 0,0 & 0,2 & 0,2 \\
\hline & 4 & 0,3 & 0,0 & 0,5 & 0,2 \\
\hline & 5 & 0,2 & 0,0 & 0,5 & 0,2 \\
\hline
\end{tabular}

Las medidas de tendencia central calculadas para la evaluación de habilidades blandas a través de la entrevista individual de competencias (tabla 7), reflejan que tanto los estudiantes del programa de psicología como los estudiantes del programa de administración en salud ocupacional cumplen con las conductas relacionadas o asociadas a la habilidad de liderazgo; la habilidad de conciliación muestra una diferencia en cuanto al promedio obtenido en los estudiantes del programa de administración en salud ocupacional y el programa de psicología, se observa que los estudiantes del programa de psicología no presentan conductas como el preguntar a participantes no activos acerca de sus opiniones, reconocer las contribuciones de otros y motivar a los demás integrantes de un equipo de trabajo para aportar más ideas.

En un sentido similar, la diferencia encontrada en la evaluación de la habilidad blanda de iniciativa en los estudiantes, evidencia que en el programa de psicología no presenta la conducta de motivar al equipo a pensar para el futuro y el ser voluntario para realizar tareas no deseadas; por el contrario, en el programa de administración de salud ocupacional, prevalece la ausencia de la conducta de proponer ideas de alta calidad. Por otra parte, se presenta una similitud en la evaluación de las conductas observables relacionadas con la habilidad de organización, ya que para ambos programas existe una ausencia de la característica o del criterio asociado a solicitar a otros a centrarse cuando se han distanciado del punto central de la discusión. Por último, en el trabajo en equipo se evalúan cuatro conductas las cuales se presentan en su totalidad en cada uno de los estudiantes que presentaron la entrevista. 
Tabla 7: Medidas de Tendencia Central de las habilidades blandas evaluadas en entrevista individual por competencias *Programa de Psicología ** Programa de Administración en Salud Ocupacional

\begin{tabular}{|c|c|c|c|c|c|c|c|}
\hline \multirow{2}{*}{ Habilidad } & \multirow{2}{*}{ Conducta } & \multicolumn{2}{|c|}{ Media } & \multicolumn{2}{|c|}{ Mediana } & \multicolumn{2}{|c|}{ Moda } \\
\hline & & $1^{*}$ & $2^{* *}$ & $1^{*}$ & $2^{* *}$ & $1^{*}$ & $2^{* *}$ \\
\hline \multirow{5}{*}{ Liderazgo } & 1 & 1 & 1 & 1 & 1 & 1 & 1 \\
\hline & 2 & 1 & 1 & 1 & 1 & 1 & 1 \\
\hline & 3 & 1 & 1 & 1 & 1 & 1 & 1 \\
\hline & 4 & 1 & 1 & 1 & 1 & 1 & 1 \\
\hline & 5 & 1 & 1 & 1 & 1 & 1 & 1 \\
\hline \multirow{5}{*}{ Conciliación } & 1 & 0 & 0 & 0 & 0 & 0 & 0 \\
\hline & 2 & 1 & 1 & 1 & 1 & 1 & 1 \\
\hline & 3 & 1 & 1 & 1 & 1 & 1 & 1 \\
\hline & 4 & 0 & 1 & 0 & 1 & 0 & 1 \\
\hline & 5 & 0 & 1 & 0 & 1 & 0 & 1 \\
\hline \multirow{5}{*}{ Iniciativa } & 1 & 1 & 1 & 1 & 1 & 1 & 1 \\
\hline & 2 & 0 & 1 & 0 & 1 & 0 & 1 \\
\hline & 3 & 1 & 0 & 1 & 0 & 1 & 0 \\
\hline & 4 & 0 & 1 & 0 & 1 & 0 & 1 \\
\hline & 5 & 1 & 1 & 1 & 1 & 1 & 1 \\
\hline \multirow{5}{*}{ Organización } & 1 & 1 & 1 & 1 & 1 & 1 & 1 \\
\hline & 2 & 1 & 1 & 1 & 1 & 1 & 1 \\
\hline & 3 & 1 & 1 & 1 & 1 & 1 & 1 \\
\hline & 4 & 0 & 0 & 0 & 0 & 1 & 0 \\
\hline & 5 & 1 & 1 & 1 & 0 & 0 & 1 \\
\hline \multirow{4}{*}{$\begin{array}{l}\text { Trabajo en } \\
\text { equipo }\end{array}$} & 1 & 1 & 1 & 1 & 1 & 1 & 1 \\
\hline & 2 & 1 & 1 & 1 & 1 & 1 & 1 \\
\hline & 3 & 1 & 1 & 1 & 1 & 1 & 1 \\
\hline & 4 & 1 & 1 & 1 & 1 & 1 & 1 \\
\hline
\end{tabular}

La desviación estándar calculada para cada una de las conductas relacionadas con las habilidades blandas evaluadas a través de la entrevista individual por competencias, muestra que no hay una diferencia representativa con relación a la media; sin embargo, es importante resaltar que las conductas alineadas a las habilidades de liderazgo, organización y trabajo en equipo no muestran desviación con relación a la media, lo cual permite inferir que los estudiantes de ambos programas académicos manifiestan conductas tales como la expresión de la confianza mediante estilos de comunicación no agresivos, el establecimiento de tareas para sí mismos y el compromiso para lograrlas hasta el final, la tolerancia con los miembros del equipo de trabajo y la aceptación de sus solicitudes o inquietudes, así como el trabajar para resolver la tarea y estar involucrados completamente en ella (tabla 8). Después de realizado los dos ejercicios, tanto el juego de roles como la entrevista individual por competencias y analizadas las conductas asociadas, se obtiene la calificación de las 10 habilidades blandas para cada programa académico (escucha, habilidad para plantear preguntas, presentación, autoconfianza, influencia, liderazgo, apoyo, iniciativa, organización y trabajo en equipo) en una escala de uno a cinco, siendo cinco la calificación más alta y uno la calificación más baja.

Las medidas de tendencia central evaluadas en el programa académico de administración en salud ocupacional aparecen en la tabla 9 y muestran que la media para la habilidad de escucha, influencia, liderazgo y trabajo en equipo es cinco (5), el valor que divide la distribución de las frecuencias por la mitad (mediana) es de cinco (5), en el caso de las habilidades de escucha, influencia, liderazgo y trabajo en equipo. La calificación más baja prevalece para este programa académico en la habilidad que tienen los estudiantes para plantear preguntas, la media de esta habilidad es de uno (1) y la mediana es dos (2) y el dato que más se repite (moda) es la calificación de uno (1) que es la más baja.

Por otra parte, La habilidad blanda enfocada al apoyo muestra una calificación promedio de tres (3), así igual que la mediana y la moda, lo cual permite identificar que para el programa académico de administración en salud ocupacional, son las dos habilidades con menor calificación. La desviación estándar para estas dos habilidades blandas con menor calificación es de 1,3 y 1,1 respectivamente, lo cual demuestra que hay una mayor dispersión en la evaluación de las conductas asociadas a las habilidades y, por ende, la heterogeneidad en los resultados de la muestra evaluada. Una vez realizada la calificación de cada una de las habilidades blandas para el programa académico de psicología tabla 10, se evidencia que ninguna de ellas muestra en su media de calificación, el valor de cinco (5) es decir, que la calificación más alta en promedio obtenido para seis de las habilidades (escucha, autoconfianza, influencia, liderazgo, organización y trabajo en equipo) fue de cuatro (4). Resulta significativo resaltar que, en las habilidades de escucha, influencia, liderazgo y trabajo en equipo, el dato que más se repite (moda) es la calificación más alta correspondiente a un valor de cinco (5); sin embargo, cuándo se evalúa el valor que divide la distribución de frecuencias por la mitad (mediana), tan sólo la habilidad de escucha, trabajo en equipo y liderazgo muestran la calificación más alta. 
Tabla 8: Medidas de dispersión de las habilidades evaluadas blandas evaluadas en entrevista individual por competencias *Programa de Psicología ** Programa de Administración en Salud Ocupacional

\begin{tabular}{|c|c|c|c|c|c|}
\hline \multirow{2}{*}{ Habilidad } & \multirow{2}{*}{ Conducta } & \multicolumn{2}{|c|}{ Varianza } & \multicolumn{2}{|c|}{ Desviación Estándar } \\
\hline & & $1^{*}$ & $2^{* *}$ & $1^{*}$ & $2^{* \star}$ \\
\hline \multirow{5}{*}{ Liderazgo } & 1 & 0,2 & 0,1 & 0,4 & 0,3 \\
\hline & 2 & 0,1 & 0,0 & 0,3 & 0,0 \\
\hline & 3 & 0,1 & 0,0 & 0,4 & 0,2 \\
\hline & 4 & 0,1 & 0,0 & 0,3 & 0,0 \\
\hline & 5 & 0,1 & 0,2 & 0,3 & 0,4 \\
\hline \multirow{5}{*}{ Conciliación } & 1 & 0,2 & 0,1 & 0,5 & 0,4 \\
\hline & 2 & 0,2 & 0,1 & 0,4 & 0,3 \\
\hline & 3 & 0,1 & 0,1 & 0,4 & 0,3 \\
\hline & 4 & 0,2 & 0,2 & 0,5 & 0,5 \\
\hline & 5 & 0,3 & 0,2 & 0,5 & 0,5 \\
\hline \multirow{5}{*}{ Iniciativa } & 1 & 0,1 & 0,1 & 0,3 & 0,3 \\
\hline & 2 & 0,3 & 0,1 & 0,5 & 0,4 \\
\hline & 3 & 0,0 & 0,3 & 0,2 & 0,5 \\
\hline & 4 & 0,2 & 0,0 & 0,5 & 0,2 \\
\hline & 5 & 0,2 & 0,1 & 0,5 & 0,4 \\
\hline \multirow{5}{*}{ Organización } & 1 & 0,1 & 0,2 & 0,3 & 0,5 \\
\hline & 2 & 0,2 & 0,2 & 0,5 & 0,4 \\
\hline & 3 & 0,0 & 0,0 & 0,2 & 0,0 \\
\hline & 4 & 0,1 & 0,2 & 0,5 & 0,5 \\
\hline & 5 & 0,0 & 0,1 & 0,2 & 0,4 \\
\hline \multirow{4}{*}{ Trabajo en equipo } & 1 & 0,2 & 0,0 & 0,4 & 0,2 \\
\hline & 2 & 0,2 & 0,0 & 0,5 & 0,0 \\
\hline & 3 & 0,2 & 0,1 & 0,5 & 0,3 \\
\hline & 4 & 0,0 & 0,0 & 0,2 & 0,0 \\
\hline
\end{tabular}

Tabla 9: Medidas de tendencia central y de dispersión de las habilidades blandas evaluadas en el programa académico de administración en salud ocupacional

\begin{tabular}{|c|c|c|c|c|c|c|c|c|c|c|}
\hline \multirow[b]{2}{*}{ Medida } & \multicolumn{5}{|c|}{ Ejercicio 1} & \multicolumn{5}{|c|}{ Ejercicio 2} \\
\hline & 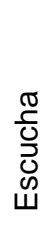 & 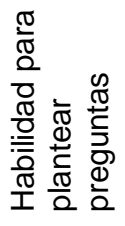 & 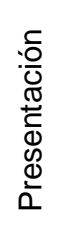 & 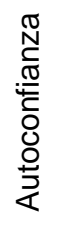 & 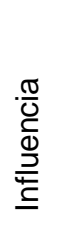 & 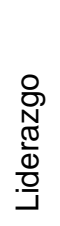 & $\begin{array}{l}0 \\
\text { Oे } \\
\frac{0}{2}\end{array}$ & 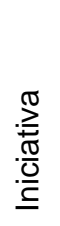 & 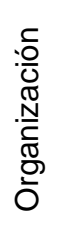 & 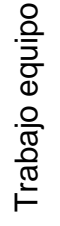 \\
\hline Media & 5 & 2 & 4 & 4 & 5 & 5 & 3 & 4 & 4 & 5 \\
\hline Mediana & 5 & 2 & 4 & 4 & 5 & 5 & 3 & 4 & 4 & 5 \\
\hline Moda & 5 & 1 & 4 & 4 & 5 & 5 & 3 & 4 & 4 & 5 \\
\hline Desviación estándar & 0,4 & 1,3 & 0,7 & 0,7 & 0,5 & 0,6 & 1,1 & 0,9 & 0,9 & 0,4 \\
\hline Varianza & 0,1 & 1,7 & 0,5 & 0,5 & 0,3 & 0,3 & 1,1 & 0,8 & 0,8 & 0,2 \\
\hline
\end{tabular}

Tabla 10: Medidas de tendencia central y de dispersión de las habilidades blandas evaluadas en el programa académico de psicología

\begin{tabular}{|c|c|c|c|c|c|c|c|c|c|c|}
\hline \multirow[b]{2}{*}{ Medida } & \multicolumn{5}{|c|}{ Ejercicio 1} & \multicolumn{5}{|c|}{ Ejercicio 2} \\
\hline & 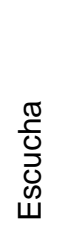 & 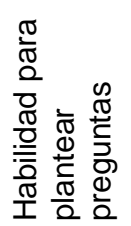 & 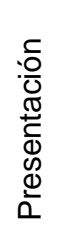 & 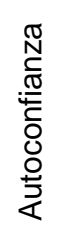 & 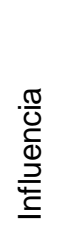 & 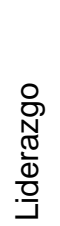 & 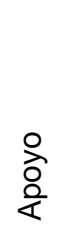 & 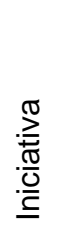 & 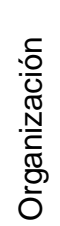 & 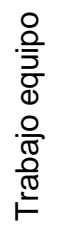 \\
\hline Media & 4 & 2 & 3 & 4 & 4 & 4 & 3 & 3 & 4 & 4 \\
\hline Mediana & 5 & 2 & 3 & 4 & 4 & 5 & 3 & 3 & 4 & 5 \\
\hline Moda & 5 & 2 & 3 & 4 & 5 & 5 & 4 & 4 & 4 & 5 \\
\hline $\begin{array}{l}\text { Desviación } \\
\text { estándar }\end{array}$ & 1,1 & 1,0 & 1,0 & 0,7 & 1,2 & 1,0 & 1,5 & 1,2 & 0,9 & 1,3 \\
\hline Varianza & 1,3 & 1,1 & 1,1 & 0,5 & 1,5 & 1,1 & 2,1 & 1,4 & 0,9 & 1,7 \\
\hline
\end{tabular}


La habilidad blanda con menor calificación en el programa académico de psicología es la relacionada con la capacidad de plantear preguntas, lo cual coincide con la evaluación realizada a las habilidades blandas en el programa académico de administración en salud ocupacional; cabe anotar que dentro de las puntuaciones bajas se encuentra la habilidad blanda de presentación, apoyo e iniciativa. La desviación estándar es mucho más alta para los resultados obtenidos en el programa académico de psicología en comparación con el programa de administración en salud ocupacional, lo cual demuestra una mayor heterogeneidad en los resultados obtenidos con relación a la media en cada una de las calificaciones de las habilidades blandas.

\section{DISCUSIÓN}

Las habilidades blandas han sido tema de principal interés por diferentes autores, es importante traer a colación a Groh et al. (2015) quién a partir de diferentes ejercicios interactivos propuso realizar la medición de las competencias en la población; la observación de las conductas de escucha, habilidad para plantear preguntas, presentación, autoconfianza, influencia, liderazgo, supportiveness (apoyo), iniciativa, organización y trabajo en equipo, fueron las principales habilidades propuestas para identificar la presencia de las mismas en los estudiantes y establecer posibles estrategias de intervención en programas de educación superior.

Una característica fundamental que se encontró en este estudio, se relaciona con la habilidad de trabajo en equipo y liderazgo, las cuáles están presentes en los estudiantes de psicología y de administración en salud ocupacional; lo cual concuerda con lo planteado por diferentes autores en el sentido que el sector empresarial demanda personas con competencias enfocadas a la orientación al conocimiento, trabajo en equipo, liderazgo, escucha (Cobo y Moravec, 2011; Tito y Serrano, 2016; Araya y González, 2019). Respecto a las habilidades de conciliación, iniciativa y organización, de acuerdo con los resultados encontrados en el estudio se observa que en los dos grupos poblacionales deben ser potencializadas en los estudiantes.

Otro aspecto interesante es la desviación estándar observada en los dos grupos de estudiantes, por cada habilidad se observa la dispersión respecto a la media: liderazgo 0,2 ; conciliación 0,4 , iniciativa 0,3 ; organización 0,4 y trabajo en equipo 0,1; escucha 0,3; habilidad para plantear preguntas 0,3 ; presentación 0,3; autoconfianza 0,2 e influencia 0,4 para el caso de los estudiantes de psicología; para los estudiantes de Administración en Salud Ocupacional se observaron los siguientes: escucha 0,1; habilidad para plantear preguntas 0,4 ; presentación 0,2; autoconfianza 0,3; influencia 0,2; liderazgo 0,2; conciliación 0,4; iniciativa 0,3 ; organización 0,3 y trabajo en equipo 0,1 . Al describir estos resultados, se evidencia homogeneidad entre los dos grupos muéstrales, por lo tanto, las habilidades blandas en los estudiantes de último semestre se encuentran presentes, aunque requieren potencializarse, ya que el grado de dispersión respecto a la media varía entre las distintas habilidades estudiadas.

De acuerdo a lo anterior, resulta interesante observar el estudio realizado por Arango, Clavijo y otros dos autores (2014) que buscaba determinar la relación entre la formación académica, la empatía, los valores y comportamientos socialmente responsables de estudiantes de primero, quinto y último semestre de varios programas académicos de la Fundación Luis Amigo Colombia. Se observó, que los niveles de empatía y comportamientos socialmente aceptables están relacionados con la formación académica, en donde aquellos programas que están orientados a la prestación de un servicio, estas habilidades tienden a estar presentes, como se evidencia en los hallazgos de este estudio.

La muestra del estudio en un $96 \%$ ha tenido experiencia laboral, este aspecto permite resaltar la presencia de estas habilidades en los estudiantes, de últimos semestres, teniendo en cuenta que los empresarios en la actualidad, buscan que los aspirantes traigan consigo habilidades socioemocionales, puesto que las habilidades duras están presentes pero las habilidades blandas deben continuar con el desarrollo a lo largo del ciclo laboral; así lo menciona Musa et al. (2012), quienes identifican la responsabilidad, confianza en sí mismos, habilidades sociales y de comunicación, flexibilidad, espíritu de equipo, buena actitud de trabajo, automotivación y autogestión como aquellas habilidades blandas que buscan los empleadores del siglo 21 en los graduados y la organización debe facilitar el proceso de continuo desarrollo (Kalugina y Shvydun, 2014). De igual manera, lo resalta Peeters et al. (2019) quienes a través de la matriz del capital de empleabilidad, consideran los recursos sociales y personales con relación al capital humano e identifican el conocimiento, las habilidades y las actitudes que dispone el empleado, así como refieren tres categorías relacionadas con la empleabilidad.

Las habilidades blandas que se desarrollan desde la formación básica, deben continuar en la educación superior, ya que facilitan el proceso de inmersión laboral de los recién egresados; sin embargo, un aspecto interesante radica en la falencia encontrada en este estudio sobre la habilidad de conciliación y de plantear preguntas. En la habilidad de conciliación tiene mayor prevalencia en los estudiantes del programa de administración en salud ocupacional, pero la habilidad de plantear preguntas está ausente en los dos grupos, característica que debe ser potencializada, ya que en su relación con la empleabilidad y el proceso de 
aprendizaje hacen parte del éxito laboral. Es así como la Organización para la Cooperación y el Desarrollo Económico [OECD], (2015) considera que la autoconfianza está relacionada con la enseñanza desde el ejemplo en edades tempranas, tanto por padres como por educadores, por lo tanto, el desarrollo de actividades curriculares de manera recurrente favorece el desarrollo de estas habilidades tanto en la educación superior como en la vida laboral.

Las habilidades de escucha y liderazgo presentan una media poblacional de 1 en los dos grupos muestrales, y una desviación estándar de 0,1 y 0,2 respectivamente, es importante aclarar que estas habilidades son necesarias para el desarrollo laboral y empleabilidad de los recién egresados, de hecho hacen parte de las competencias que en el Siglo XXI se busca en los profesionales, tanto directivos como operativos y están relacionados con el desarrollo de carrera y aprendizaje; se observa una gran participación para potencializar estas habilidades en la educación superior como lo afirma Busso et al. (2012) quienes afirman la importancia de generar estrategias educativas que favorezcan la igualdad y equidad en los procesos formativos. De acuerdo a lo anterior, es preciso afirmar que la educación superior en el desarrollo de habilidades blandas y duras cumplen una función principal, el fortalecimiento de estrategias y la preparación de los futuros profesionales depende del quehacer educativo en su formación no sólo teórica, también en habilidades éticas y socioemocionales que permitan potencializar competencias para lograr objetivos personales y empresariales, con el fin de tomar decisiones acertadas, responsables y autónomas que permitan el buen relacionamiento y la construcción colectiva de procesos organizacionales exitosos (Berry y Routon, 2020).

El Siglo XXI busca que los profesionales cuenten con competencias de pensamiento estratégico, pensamiento sistémico, toma de decisiones, apertura al cambio, ética, liderazgo, comunicación entre otras, ante un mundo cambiante y competitivo en el cual laboralmente no solo prima el conocimiento y la relación teoría Vs práctica; también debe primar el trabajo en equipo, capacidad de escucha, capacidad influenciadora, iniciativa y organización, que permitan el logro de objetivos no solo personales sino empresariales, es así como el manejo de emociones y los procesos de socialización deben marcar la diferencia en los escenarios educativos y organizacionales, tanto en niveles operativos como directivos y que estos permitan la estabilidad y la inmersión laboral en profesionales recién egresados y con poca experiencia laboral relacionada con el perfil ocupacional.

La educación superior tiene el compromiso de crear estrategias de aprendizaje y desarrollo continuo de habilidades socioemocionales, en el cual se contribuya a mejorar las competencias de los futuros profesionales y prepararlos ante un mundo cambiante, competitivo y dinámico en el cual prevalezca la ética, el trabajo en equipo, el liderazgo y la comunicación, como habilidades principales en los procesos de selección y empleabilidad. Si bien es cierto que las habilidades observadas en este estudio están homogéneas en los dos grupos muestrales, se hace necesario potencializarlas por medio de estrategias educativas en el aula que permitan el desarrollo latente y la presencia de las mismas en todos los estudiantes de últimos semestres. En próximas investigaciones sería conveniente analizar estudiantes de diferentes carreras de últimos semestres, con el fin de evaluar la presencia y desarrollo de diferentes estrategias educativas que promuevan la vinculación laboral de los recién egresados en empresas de diferentes sectores económicos.

\section{CONCLUSIONES}

De acuerdo al trabajo presentado y a los resultados obtenidos, se pueden plantear las siguientes conclusiones principales:

1. Las habilidades blandas se consideran un eje fundamental en el proceso de empleabilidad, y por tal motivo se considera que las instituciones de educación superior deben adoptar y evaluar procesos de enseñanza aprendizaje que se articulen con la potencialización de las mismas.

2. Los estudiantes tienen una gran responsabilidad con el desarrollo de estas habilidades, ya que muchas de ellas se desarrollan desde su primera infancia; y por lo tanto, es imperativo que se reconozca el valor agregado que estas pueden generar en su currículo.

3. La gran mayoría de los estudiantes de ambos programas, muestran déficit en la habilidad para hacer preguntas, lo cual se relaciona directamente con los procesos de comunicación, y por tal motivo se encuentra relevante generar estrategias que potencien esta habilidad en los futuros egresados.

4. Se evidencia la prevalencia del $90 \%$ de las habilidades no cognitivas evaluadas en los sujetos de estudio, comprendiendo este conjunto las habilidades de escucha, presentación, autoconfianza, influencia, liderazgo, apoyo, iniciativa, organización y trabajo equipo. 


\section{REFERENCIAS}

Araya, E., y González, G., Propuesta para el fortalecimiento de habilidades técnicas, blandas y complementarias, y su impacto en el currículo TIC desde una perspectiva laboral, profesional y de gestión académica, http://dx.doi.org/10.22458/caes.v10i2.1907, Revista Electrónica Calidad en la Educación Superior, 10 (2), 112-14, (2019).

Arango, O.E., Clavijo, S.J., y otros dos autores, Formación académica, valores, empatía y comportamientos socialmente responsables en estudiantes universitarios, Revista de la Educación Superior, 43(169), 89-105 (2014).

Berry, R. y Routon, W., Soft Skill Change Perceptions of Accounting Majors: Current Practitioner Views Versus their Own Reality, https://doi.org/10.1016/j.jaccedu.2020.100691, Journal of Accounting Education, 53, 1-12 (2020).

Busso, M., Bassi, M., Urzúa, S., y Vargas, J. Desconectados: habilidades, educación y empleo en América Latina. Banco Interamericano de Desarrollo (2012).

Buxarrais, M. Nuevos valores para una nueva sociedad. Un cambio de paradigma en educación. Edetania, (43), 53-65. (2013).

Cobo, C., y Moravec, J.W., Aprendizaje Invisible hacia una nueva ecología de la educación, 1ª Ed., 1-239, Collecció Transmedia XXI, Barcelona, España, (2011).

Espinosa, M.A., y Gallegos, D., Habilidades blandas en la educación y la empresa: mapeo sistemático, https://doi.org/10.35290/rcui.v7n2.2020.245, Revista Científica Uisrael, 7(2), 39-56, (2020).

Gómez, M., Las habilidades blandas competencias para el nuevo milenio, https://doi.org/10.29057/esa.v6i11.3760, Divulgare Boletín Científico de la Escuela Superior de Actopan, 6(11), 1-5 (2019).

Groh, M; Mckenzie, D, y Vishwanath, T. Reducing Information Asymmetries in the Youth Labor Market of Jordan with Psychometrics and Skill Based Tests, https://doi.org/10.1093/wber/lhv005, The World Bank Economic Review, 29, S106-S117, (2015).

Kalugina, E. y Shvydun, S., An Effective Personnel Selection Model, https://doi.org/10.1016/j.procs.2014.05.365, Procedia Computer Science, 31, 1102-1106 (2014).

OECD, Skills for Social Progress: The Power of Social and Emotional Skills, OECD Skills Studies, https://doi.org/10.1787/9789264226159-en, (2015).

Morales-Maure, L., García-Marimón, O., y otros dos autores, Habilidades cognitivas a través de la estrategia de aprendizaje cooperativo y perfeccionamiento epistemológico en matemática de estudiantes de primer año de universidad, http://dx.doi.org/10.4067/S0718-50062018000200045, Form. Univ, 11(2), 45-56 (2018).

Musa, F., Mufti, N., L, R.A.,y Amin, M.M., Project-Based Learning (PjBL): inculcating soft skills in $21^{\text {st }}$ century workplace, https://doi.org/10.1016/j.sbspro.2012.09.315, Procedia Social and Behavioral Sciences, 59, 565-573 (2012)

Musicco, G. Las soft skills \& coaching: motor de la Universidad en Europa. RUE: Revista Universitaria Europea, (29), 115-132. (2018)

Ortega, T. Desenredando la conversación sobre habilidades blandas. Informe de Educación, Banco de Desarrollo de América Latina. (2016)

Ortega, C., Febles, J., y Estrada, V., Una estrategia para la formación de competencias blandas desde edades tempranas, Revista Cubana de Educación Superior, 35(2), 35-41 (2016)

Prada, M. F., Rucci, G., y Urzúa, S., Training, soft skills and productivity: evidence from a Field Experiment in Retail, http://dx.doi.org/10.18235/0001714, Inter-American Development Bank, IDB working paper series (1015), 1-53 (2019)

Peeters, E., Nelissen, J., y otros 4 autores, Employability Capital: A Conceptual Framework Tested Through Expert Analysis, https://doi.org/10.1177/0894845317731865, Journal of Career Development, 46(2), 79-93 (2019)

Runciman, B., IT Leaders Report: Cloud, Security \& Soft Skills Come Into Their Own, https://doi.org/10.1093/itnow/bwab001, ITNOW, 63(1), 6-9 (2021)

Tito, M., y Serrano, B., Desarrollo de soft skills una alternativa a la escasez de talento humano, https://doi.org/10.33890/innova.v1.n12.2016.81, INNOVA Research Journal, 1(12), 59-76 (2016)

Tseng, H., Yi, X., y Yeh, H. T., Learning-related soft skills among online business students in higher education: Grade level and managerial role differences in self-regulation, motivation, and social skill, https://doi.org/10.1016/j.chb.2018.11.035, Computers in Human Behavior, 95, 179-186. (2019)

Zhang, A., Peer Assessment of Soft Skills and Hard Skills, Journal of Information Technology Education: Research, 11, $155-168,(2012)$ 